\title{
Attentional processing of "unattended" flankers: Evidence for a failure of selective attention
}

\author{
PATRICIA A. SCHMIDT and VERONICA J. DARK \\ Iowa State University, Ames, Iowa
}

\begin{abstract}
Results from research with the flanker task have been used to argue both that flankers are identified without attention and that flanker identificatin requires attention. In three experiments, we addressed this issue by examining flanker recall. In Experiment 1, we manipulated flanker redundancy, a variable that could influence attention to the flankers, in order to determine whether it affected the magnitude of the flanker effect, the magnitude of flanker recall, or both. Redundancy did not influence the flanker effect, and recall was high in both conditions, suggesting that the flankers were attended. The recall results contradicted those of Miller (1987), the only other study that we could find in which flanker recall was used. In Experiments 2 and 3, we examined differences between Miller's procedure and ours. Although flanker recall was lower when open-ended rather than forced recall was used and when the flanker task was made more complicated, flanker recall remained well above chance in all conditions. The data strongly suggest that in the typical correlated flanker task there is a failure of selective attention at some point during processing such that flankers receive attentional processing.
\end{abstract}

Dual-process theories (see, e.g., Cowan, 1988; Hoffman, 1979; Jonides, 1981; Logan, 1988; Neumann, 1984; Posner \& Snyder, 1975; Shiffrin \& Schneider, 1977; Treisman \& Gelade, 1980) make a clear theoretical distinction between automatic and attentional processing. Specifically, automatic processing is purported to precede attentional processing (in several models, it is the selective attention mechanism that selects some of the stimuli at the automatic level to continue to the attentional level) and to have these characteristics: It (1) is unlimited in capacity, (2) is effortless, (3) is involuntary, and (4) occurs outside of awareness (e.g., an individual may not have conscious knowledge of its progression). Attentional processing occurs after automatic processing and is purported to have these characteristics: It (1) is limited in capacity, (2) is effortful, (3) is under the control of the individual (i.e., it is flexible and influenced by intention), and (4) is more closely associated with conscious awareness. In fact, models of memory assume that attentional processing is a prerequisite for the formation of an explicit long-term memory of an event (see, e.g., Logan, 1988; Roediger, 1990; Shiffrin \& Schneider, 1977).

In the dual-process literature, a question of continuing interest, often referred to as the early-versus late-selection controversy, is whether automatic processing includes stimulus identification or whether stimulus identification

We thank Elizabeth Andre, Karla Benson, Shawna Caruthers, Amy Darda, Amanda Easton, Laura Held, Jodee Huisman, Jeannette MerrillRoth, Brian Sheesley, and Rob Such for help with data collection. Portions of the data were presented at the meeting of the Iowa Academy of Science in Decorah, April 1993. Requests for reprints can be sent to either P. A. Schmidt or V. J. Dark, Department of Psychology, lowa State University, Ames, IA 50011-3180 (e-mail: pas@iastate.edu or vjdark (1astate.edu). is related to the limited-capacity processing associated with attention (e.g., Broadbent, 1958; Deutsch \& Deutsch, 1963; Kahneman \& Treisman, 1984; for reviews see Holender, 1986; Johnston \& Dark, 1986; Lambert, 1985). Because automatic processing occurs outside of awareness, its properties are examined indirectly via the influence that automatically processed stimuli have on a response that requires attentional processing. The logic of the research is that if a response to an attended stimulus is influenced by some characteristic of an automatically processed stimulus, that characteristic must be part of automatic processing.

Researchers addressing the nature of automatic processing often have used focused-attention tasks in which stimuli in some locations are to be attended and stimuli in other locations are to be ignored. The flanker task, which we employed in the present research, is such a task. In the typical flanker task, a single target letter is presented surrounded by distractor letters, or flankers (see, e.g., B. A. Eriksen \& C. W. Eriksen, 1974; C. W. Eriksen \& Schultz, 1979; Flowers \& Wilcox, 1982; Miller, 1987; Paquet \& Lortie, 1990). The flankers are to be ignored, and the target is to be attended and requires a response. Usually, the response is binary; that is, each target is assigned to one of two response sets. After learning which targets are assigned to which response, participants are presented displays consisting of a row of letters with a target in the center and flankers to the right and left of the target or a circular arrangement of letters with flankers that are adjacent to the target. The task is to classify the target, by making the appropriate response, while ignoring the flankers. The typical outcome is that the identity of the flankers affects the speed, the accuracy, or both the speed and the accuracy of the target response (B. A. Eriksen \& C. W. Eriksen, 1974), a phenomenon referred to as the flanker effect. 
Two very different theoretical interpretations of the flanker effect are found in the literature. One is that the flanker effect is the result of automatic processing and, thus, is a demonstration of the fact that automatic processing includes stimulus identification (e.g., Evans \& Craig, 1992; Cowan, 1988; Miller, 1987, 1991; Shiffrin, Diller, \& Cohen, 1996). For example, Miller (1987) states that the flanker effect "provides an example of semantic processing of unattended stimuli" (p. 419). Likewise, Shiffrin et al. (1996) state that "the flanker studies ... provide perhaps the major line of evidence for mandatory, automatic processing of information from locations other than those to which attention is directed" (p. 226). This interpretation depends on what we call the intention-equalsattention view of processing in the flanker task. In this view, stimuli are defined as being "attended" or "unattended" in terms of participant intention. Intention can be conceived of as a conceptually driven attentional mechanism that allows one to "direct" attentional resources to various stimuli (Logan, 1995). In the flanker task, the target is labeled to be attended and the flankers are labeled to be ignored. By the intention-equals-attention view, because participants are instructed to ignore the flankers, it is assumed that the flankers are successfully excluded from attentional processing. Any influence that the flankers have on target processing must, therefore, reflect automatic processing. The accuracy of this interpretation has been accepted by some in the literature with the result that the existence of the flanker effect is cited in support of the position that identity is part of automatic processing (e.g., Cowan, 1988; Miller, 1987; Shiffrin et al., 1996).

The alternative interpretation of the flanker effect is that it demonstrates a failure of selective attention rather than identification of automatically processed stimuli (see, e.g., Chastain, Cheal, \& Lyon, 1996; Holender, 1986; Johnston \& Dark, 1986; Lambert, 1985; McCann, Folk, \& Johnston, 1992; Paquet \& Lortie, 1990; Yantis \& Johnston, 1990). The underlying idea is that intention cannot define attention, because a participant's intention to attend only to the target might be insufficient to restrict attentional processing to just the target (cf. the automatic attention response of Shiffrin \& Schneider, 1977). Much of the research supporting this view has used the following logic: Some variable is manipulated that is assumed to affect the ease of target selection and, therefore, the amount of attentional processing received by the flankers. If the magnitude of the flanker effect varies systematically with the manipulation, it is then concluded that the flanker effect depends on attentional processing of the flankers. In fact, Eriksen and his colleagues (B. A. Eriksen \& C. W. Eriksen, 1974; C. W. Eriksen \& Hoffman, 1973; C. W. Eriksen \& Schultz, 1979) actually interpreted the flanker effect as evidence for a limitation of focused attention. They suggested that attention could not be focused more narrowly than $1^{\circ}$ of visual angle and pointed out that flankers are typically presented within $1^{\circ}$ of visual angle of the target. If a failure of selective attention is the typical outcome in the flanker task so that flankers really are "attended," conclusions about the nature of automatic processing (e.g., whether it includes stimulus identification) based on the flanker task are unwarranted.

The literature thus contains contradictory interpretations of the flanker effect. The same flanker effect has been interpreted as showing support both for automatic stimulus identification and for stimulus identification that depends on a failure of selective attention. The problem with distinguishing between these two views is that one must know how to map the theoretical distinction between automatic and attentional processing to the empirical description of a stimulus as either attended or unattended. Difficulty determining just when stimuli are unattended necessarily creates ambiguity in determining the nature of automatic processing (see Holender, 1986, for a review of many of the problems). What is needed, then, is a measure of attendedness that is acceptable to researchers advocating both interpretations of the flanker effect. The solution explored in the present research was to examine explicit memory for the flankers' identities.

The relationship between processing and explicit longterm memory (the type that can be assessed with a direct memory test like recall) marks a key distinction between automatic and attentional processing for which there is good agreement in the literature. As already stated, it is assumed that formation of explicit long-term memories requires attentional processing (see, e.g., Eich, 1984; Fisk \& Schneider, 1984; Rock \& Gutman, 1981; Roediger, 1990; Shiffrin \& Schneider, 1977). Thus, explicit long-term memories will not be formed on the basis of automatic processing. For this reason, recall can be used as an imperfect measure of stimulus attendedness: If a stimulus can be recalled, it has been attended. Following this logic, one can assume that if a person is able to recall to-be-ignored stimuli, like the flankers, then the flankers must have received attentional processing. That is, a failure of selective attention must have occurred. ${ }^{!}$

Miller (1987, Experiment 4) followed a similar line of reasoning when he assessed flanker recall and participants' awareness of the relationship between the flankers and responses in a correlated flanker task. Although there was a reliable flanker effect, Miller found no evidence that participants could report the identity of the flankers or could describe the relationship between flankers and targets. Miller (1987) interpreted his finding of a flanker effect in the absence of flanker recall as evidence that the flankers were unattended and that automatic processing includes stimulus identification.

Although the relationship between explicit memory and attendedness can allow one to begin empirically to distinguish between automatic and attentional processing, the underlying causal logic is strongly one-sided: Recall implies attentional processing, but lack of recall does not imply just automatic processing. This is because factors other than the existence of an explicit memory trace (as a result of attention) affect recall (e.g., retrieval failures; see Tulving, 1983). Therefore, to use recall as a measure of attendedness produces more convincing evidence when 
recall is found than when it is not. In a sense, finding no recall is an ambiguous outcome. It could mean that the stimulus did not undergo attentional processing, but it could mean that some other factor was interfering with access to an available memory.

To our knowledge Miller (1987, Experiment 4) has been the only investigator to use recall to assess flanker attendedness. Although his argument based on a lack of recall is not as logically convincing as one based on finding recall, if further attempts to find evidence of flanker recall also fail, the case becomes more convincing. For that reason, we thought it important to attempt to replicate Miller's finding of no flanker recall, and we did so in three experiments. In Experiment 1, we manipulated flanker redundancy, a variable that could influence attention to the flankers, in order to determine whether it would affect the magnitude of the flanker effect, the magnitude of flanker recall, or both. Redundancy did not influence the flanker effect, and recall was high in both conditions, contradicting the findings of Miller (1987, Experiment 4). In Experiments 2 and 3, we examined differences between Miller's procedure and ours. Flanker recall remained above chance in all conditions, but it was lower when more Miller-like procedures were followed.

\section{EXPERIMENT 1}

A number of display characteristics assumed to enhance focused attention (e.g., target-flanker separation, similarity, precuing) have been shown to affect the magnitude of the flanker effect. The magnitude of the flanker effect is decreased as a function of manipulations that have seemed to increase the ease of target selection, suggesting that as target selection becomes easier, the likelihood of a failure of selective attention is lessened, and the effect of the flankers is reduced (e.g., Baylis \& Driver, 1992; B. A. Eriksen \& C. W. Eriksen, 1974; C. W. Eriksen \& Hoffman, 1973; C. W. Eriksen \& Schultz, 1979; Flowers, 1990; Flowers \& Wilcox, 1982; Harms \& Bundesen, 1983; Kramer \& Jacobson, 1991; Miller, 1991; Paquet \& Lortie, 1990; Stadler \& Proctor, 1993; Taylor, 1977; Yantis \& Johnston, 1990). This possibility would be supported by evidence that participants were able to recall the to-beignored flankers under conditions of difficult, but not easy, target selection. In Experiment 1, we manipulated a display characteristic (flanker redundancy) that might affect the ease of target selection, and we assessed participants' ability to recall the letters that served as flankers.

We were interested in whether or not flanker redundancy contributes to the magnitude of the flanker effect. Flanker redundancy refers to the stimulus configuration whereby the same flanker letters surround a target. In the typical flanker study, the stimulus displays are arranged so that identical flankers-that is, the same letters-are placed to the right and left of the target. This letter configuration creates a symmetric envelope that surrounds the target. Hence, in the present research, redundant flanker displays were created by placing the same flanker to the right and left of the target. Nonredundant flanker displays were created by placing a different but equally predictive (i.e., it was associated with the same response) flanker to the right and left of the target. One question was whether a disruption of flanker redundancy would lead to a reduction in the magnitude of the flanker effect. Further, following the flanker recall logic previously outlined, a second question was whether a disruption of flanker redundancy would affect whether the flankers were attended (i.e., whether flanker recall would be high).

Researchers examining the impact of redundant targets have shown that stimulus redundancy can facilitate the processing of targets (e.g., Fournier \& C. W. Eriksen, 1990; Miller, 1982); that is, responses to a target are faster when that target is repeated within the display than when it is displayed in the presence of a neutral stimulus. The same may be true of flankers. Flanker redundancy may actually lead to a failure of selective attention if automatic processing of two identical flankers coactivates them so that they surpass the threshold for attentive processing. If coactivation from the two identical flankers leads to the failure of selective attention and this is what leads to the flanker effect, then disruption of the redundancy, which can be accomplished by using nonredundant flankers, should reduce or eliminate the effect of the flankers on target processing. Another prediction from this line of reasoning is that the disruption in the flanker effect should be accompanied by a reduction in flanker recall.

In Experiment 1, we used Miller's (1987) correlated flanker task, which was originally developed because data from the Eriksen flanker task are open to the criticism that the processing of the flankers is facilitated by priming (because the letters are task relevant when they occur in the target location). The correlated flanker task differs from the Eriksen flanker task in that targets and flankers are consistently mapped to location (cf. Shiffrin \& Schneider, 1977). Specifically, whereas with the Eriksen flanker task the same letters serve as both targets and flankers on different trials, with the correlated flanker task the flankers never serve as targets and the targets never serve as flankers.

The correlated flanker task allows flankers to become associated with one or the other response without being explicitly assigned to that response: Flankers are never targets, but specific flankers co-occur regularly with targets requiring a specific response. That is, a flanker-response correlation is present. The response-correlated flankers become associated with one or the other response and come to influence target responses (i.e., there is a flanker effect). Although similar results have been found with both the Eriksen flanker task and the correlated flanker task (e.g., Miller, 1987; Paquet \& Lortie, 1990), we adopted the correlated flanker task for the present research because it allowed us to obtain a measure of flanker recall for flankers that participants were not required to learn as part of the task.

The purpose of Experiment 1 was threefold: First, we were interested in exploring the effect of flanker redundancy on the magnitude of the flanker effect. Second, we were interested in replicating or not replicating Miller's 
Table 1

Example of Redundant and Nonredundant Displays as a Function of Trial Validity and Response

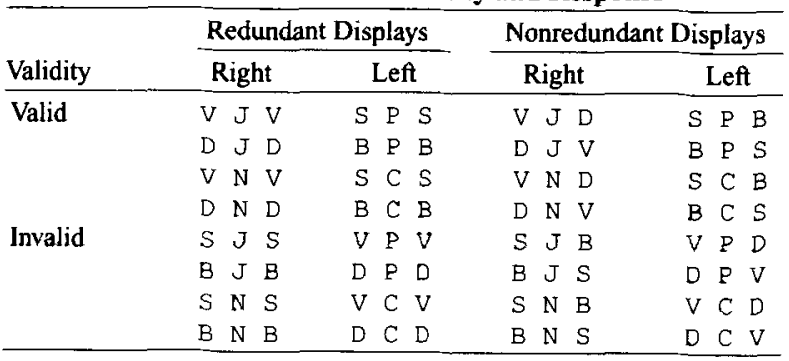

Note- In this example, $J$ and $N$ are right-hand response targets, and $P$ and $\mathrm{C}$ are left-hand response targets. Valid trials comprised $80 \%$ of total trials, and invalid trials comprised $20 \%$ of total trials.

(1987) finding of no flanker recall under conditions of redundant (and perhaps nonredundant) flankers. Third, we were interested in the relationship between the flanker effect and flanker recall. A pattern showing no evidence of flanker recall but evidence of a flanker effect, at least in the redundant flanker condition, would strengthen Miller's interpretation of the flanker effect. A pattern showing a positive relationship between the magnitude of the flanker effect and the magnitude of flanker recall would strengthen the interpretation of the flanker effect as resulting from a failure of selective attention.

\section{Method}

Participants. Forty-eight students ( 25 female and 23 male) received extra credit in an undergraduate psychology course for their voluntary participation in this experiment.

Stimuli. The stimuli were strings of three letters, chosen from the standard set of alphanumeric characters, presented in a row in the center of a VGA computer monitor. A blank space separated each letter. Letters were approximately $4 \mathrm{~cm}$ wide and $.5 \mathrm{~cm}$ high, so that when viewed from $60 \mathrm{~cm}$ the three-letter displays covered about $2^{\circ}$ of visual angle. Each flanker was within $1^{\circ}$ of visual angle of the target (although this may have varied slightly by participant because no chinrest was used).

Each participant was randomly assigned eight letters from the consonants B-W. Two of these letters served as targets in the righthand response set, two of them served as targets in the left-hand response set, and the remaining four were used in the flanker positions. Letters that were chosen as targets never appeared as flankers and vice versa. Flanker validity (i.e., the flanker-response correlation) was .80. That is, two of the four flankers appeared with right-hand targets on $80 \%$ of the experimental trials (valid trials) and with the left-hand targets on $20 \%$ of the experimental trials (invalid trials). The other two flankers appeared with left-hand targets on $80 \%$ of the experimental trials and with right-hand targets on $20 \%$ of the trials. In each redundancy condition, there were eight different valid displays and eight different invalid displays.

Flanker redundancy was manipulated by varying which flankers surrounded a target. Table 1 shows a sample set of stimulus displays. Redundant stimulus displays were constructed by presenting a target from one of the response sets with the same flanker letter to the right and left of it. Nonredundant stimulus displays were constructed by presenting a target from one of the response sets with a different flanker to the right and left of it. On a given nonredundant trial, the two different flankers surrounding the target were always associated with the same response.
Procedure. Stimulus presentation and recording of response latency was controlled by a Zenith-386 computer programmed using the MicroExperimental Laboratory (MEL) software (Schneider, 1988).

Participants were assigned to either the redundant or nonredundant flankers condition and were tested individually in a single session. There were six blocks of trials in each redundancy condition. The first block, which was practice, consisted of 32 trials in which flanker validity was 1.00 (i.e., there were no invalid trials). Each of the valid displays was presented four times, ensuring that there was opportunity to begin learning the flanker-response correlations. The second through fifth biocks were the experimental blocks on which reaction times to valid and invalid trials were measured. Each block contained 32 right-hand response trials with valid flankers, 32 lefthand response trials with valid flankers, 8 right-hand response trials with invalid flankers, and 8 left-hand response trials with invalid flankers. Therefore, each experimental block consisted of 80 trials over which the flanker validity was .80 . There were 320 experimental trials total.

Each trial began with a 1,000-msec "READY" signal and a target location cue $(\wedge)$ that appeared below the "A" in "READY." The location cue indicated where the target would occur. The "READY" signal was followed by a 500 -msec delay in which only the location cue was present. Finally, the target location cue remained on the computer screen while the stimulus display was presented. The entire stimulus display (viz., a target surrounded by flankers with a target location cue directly below the target) remained on the computer screen until the participant made a response or a $1,500-\mathrm{msec}$ response deadline expired.

Participants learned the two right-hand response targets and the two left-hand response targets. Participants were instructed to attend to the target letter that appeared above the target location cue $\left({ }^{\wedge}\right)$ and to ignore the flanking letters that were presented to the right and left of the target letter on each trial. Responses were made by pressing the "/" key with the right index finger for right-hand response targets and by pressing the " $Z$ " key with the left index finger for the left-hand response targets. Participants rested their index fingers on the "/" and " $Z$ " keys throughout the experiment to help speed their responses. Error feedback was presented immediately: Incorrect responses to targets and failures to respond within $1,500 \mathrm{msec}$ were signaled by a $500-\mathrm{msec}, 400-\mathrm{Hz}$ tone; no tone occurred following responses that were correct and within the time limit. The intertrial interval was $1,000 \mathrm{msec}$, during which time the computer screen was blank. A participant-terminated rest period occurred between each of the experimental blocks.

After the five blocks of experimental trials, participants were required to verbally report the four target letters and the four flanker letters. The request for flanker recall was preceded by a statement informing the participants that although they had been instructed to ignore the flankers, they might have noticed them during the trials and that the research was designed to assess the extent to which that occurred. That is, an attempt was made to legitimize correct report of the flankers. The participants were instructed to report four letters as flankers even though they might not be certain about their responses. The proportion of accurate responses defined flanker recall.

After target and flanker recall, the sixth block of (32) trials was presented, in which novel symbols appeared in the target position with the same flankers that surrounded the letter targets in the experimental trials. The sixth block was designed to assess implicit knowledge of the flanker-response relationships, so the participants were instructed to make the response they "felt" was appropriate to these displays. ${ }^{2}$ Following this block, explicit knowledge of the flanker-response relationships was assessed. The participants were required to again report four flanker letters and to indicate which two flankers they believed were usually presented with the righthand response targets and which two flankers they believed were usually presented with the left-hand response targets. That is, the 
Table 2

Mean Latency (in Milliseconds) With Standard Deviations

and Proportions Correct for Valid and Invalid Trials and the

Flanker Effect as a Function of Redundancy for Experiment 1

\begin{tabular}{lccccccc} 
& \multicolumn{3}{c}{ Redundant } & & \multicolumn{3}{c}{ Nonredundant } \\
\cline { 2 - 3 } \cline { 6 - 8 } \multicolumn{1}{c}{ Validity } & $M$ & $S D$ & PC & & $M$ & $S D$ & PC \\
\hline Valid & 515 & 61 & .98 & & 505 & 37 & .97 \\
Invalid & 527 & 72 & .98 & & 521 & 38 & .97 \\
Flanker effect & 12 & 17 & & 16 & 14 & \\
\hline
\end{tabular}

Note- - Flanker effect $=$ invalid latency - valid latency .

participants were asked to assign the flankers to one of the responses. The proportion of accurate responses defined flanker assignment. Finally, all participants were debriefed before the experimental session was terminated. The average time for a session was $35 \mathrm{~min}$.

\section{Results}

An alpha level of .05 was used for all analyses. The data from 2 participants were replaced because their accuracy fell below our $90 \%$ criterion.

Flanker effect. The latency analysis included accurate response latencies greater than $50 \mathrm{msec}$. The mean latencies with standard deviations and proportion correct responses are shown in Table 2 as a function of trial validity (invalid or valid) for the redundant and nonredundant conditions. The flanker effect, computed as the difference in latency between invalid and valid trials, is shown in the bottom row of Table 2 . Accuracy was $96 \%$ or higher in all conditions, with no evidence of any speed-accuracy tradeoffs. An analysis of variance (ANOVA) of the latency data with validity as a within-subjects variable and redundancy condition as a between-subjects variable revealed only a main effect of validity $\left[F(1,46)=36.36, M S_{\mathrm{e}}=127\right]$. Latencies to invalid trials were reliably longer than latencies to valid trials. There was no main effect of redundancy and no interaction, indicating that the magnitude of the flanker effect was similar for both redundancy conditions. That is, the flanker effect was reliable and did not depend on flanker redundancy.

Flanker recall. The mean proportion of flankers correctly recalled was calculated by dividing the number of flankers correctly identified by four, the number of flankers to be identified. The mean proportions were $0.71(S D=$ $.26)$ and $0.74(S D=.25)$ for the redundant and nonredundant conditions, respectively. Chance level flanker recall was calculated to determine guessing rates. We used a hypergeometric distribution (nonindependent cases) to determine the probability of guessing $0,1,2,3$, or 4 of the 4 possible flankers. These calculations yielded an expected value of .73 of 4 or a flanker recall proportion of . 18 by chance alone. No attempt was made to correct for the differential guessing rates associated with various letters, because each participant viewed a randomly selected set of letters. One-tailed tests showed that flanker recall was higher than would be expected by chance both for the redundant condition $[t(23)=9.81, S E=0.054]$ and for the nonredundant condition $[t(23)=10.98, S E=0.051]$. There was no reliable difference in the level of flanker re- call between the two redundancy conditions $[t(46)=0.41$, $S E=0.074]$.

Flanker assignment. Because participants had already attempted recall of flankers and had been exposed to the flankers again during the sixth block, accuracy of recall of the flankers was somewhat higher during flanker assignment than during original recall (.82 vs. .72$)$. The mean proportion of flankers assigned to the correct response was calculated by dividing the total number of flankers correctly assigned to responses by the total number of flankers correctly reported during flank assignment. The mean proportions were $.36(S D=.31)$ and $.46(S D=$ .49) for the redundant and nonredundant conditions, respectively. Chance level flanker assignment would be .50 . In neither redundancy condition was flanker assignment reliably higher than chance with a one-tailed test ${ }^{3}[t(23)=$ $-2.26, S E=0.062$, for the redundant condition, and $t(23)=-0.40, S E=0.099$, for the nonredundant condition]. There was no reliable difference in the level of correct flanker assignment between the two redundancy conditions $[t(46)=0.83, S E=0.117]$. Thus, although the participants appeared to be aware of flanker identities, there was no evidence that they had any explicit knowledge of which flankers went with which response.

\section{Discussion}

Experiment 1 was designed to test the impact of flanker redundancy on both the flanker effect and flanker recall. Flanker redundancy did not affect the magnitude of the flanker effect. Thus, there was no support for the hypothesis that the flanker effect is the result of a failure of selective attention that occurs because of coactivation of flanker representations. However, the flanker recall results showed that there was a failure of selective attention at some point during processing for both redundant and nonredundant displays. For both types of display, flankers were identified at a level well above that expected by chance. This finding suggests that participants were aware of the flanker letters. The results from the flanker assignment analysis indicated, however, that participants were not aware of which flankers were correlated with which response. Thus, although the present results show that the flanker effect is associated with knowledge of flanker identities, it does not appear to require explicit knowledge of the flanker-response relationships.

Miller (1987) stated that a high level of flanker recall would be evidence that the flankers underwent attentional 
processing, but when Miller assessed flanker recall and also participants' awareness of the flanker-response correlations, he found no evidence that participants could recall the flanker letters or could describe the relationship between flankers and target responses. He concluded that his findings were inconsistent with appeals to a failure of selective attention in explaining the flanker effect. Further, Miller suggested that the flanker effect showed that there was semantic processing of unattended stimuli; it was flanker identity that predicted the appropriate target response, so flanker identity must be preattentively available.

Miller's (1987) conclusions rest squarely on the failure to find any evidence of flanker recall. Although the flanker assignment data of Experiment 1 replicated Miller's finding that participants are unaware of the flanker-response relationships, the recall data were inconsistent with those reported by Miller (1987): Very high flanker recall was found in both redundancy conditions. Participants appeared to process both the targets and the flankers attentionally; that is, there appeared to be a failure of selective attention at some point during processing with both redundant and nonredundant flankers.

Miller (1987, Experiment 4) was the only study that we could find in which flanker recall was assessed. Miller's results and ours do not agree. Given the discrepancy and the apparent importance of flanker recall (or the lack thereof) to interpretations of the flanker effect, Experiment 2 was designed to assess whether there might be differences in the sensitivity between Miller's recall measure and ours.

\section{EXPERIMENT 2}

In Experiment 2, we investigated the discrepancy between the flanker recall findings in Experiment 1 and those reported by Miller (1987). We first compared the general display parameters and procedure used by Miller in the fully correlated condition of his Experiment 4 and in the present Experiment 1. There appeared to be few meaningful differences. ${ }^{4}$ We and Miller both used computer displays with simultaneous flanker and target onsets, used randomly selected different letters for each participant, and presented displays until a response was made or a relatively long response deadline expired $(10 \mathrm{sec}$ for Miller and 1,500 msec for Experiment 1). We did, however, use a target location cue, whereas Miller did not use a target location cue in the condition in which he assessed flanker recall. Also, we differed in the degree of flanker validity employed: We used a .80 flanker validity, whereas Miller used a .92 flanker validity.

We next compared the recall procedures. Our assessment of flanker recall in Experiment 1 differed from Miller's (1987) in at least two ways that may have increased the sensitivity of our recall measure. First, we made an explicit attempt to legitimize recall. It is unclear whether or not Miller made an attempt to legitimize the task to participants. Miller's participants may have been influenced by the initial instructions to ignore the flankers and may have chosen not to report the flankers for which a mem- ory trace was both available and accessible. Second, we required participants to name four letters as flankers, whereas Miller did not. If Miller's participants adopted a strict response criterion for flanker recall as a result of the experimental instructions, they may have chosen to withhold responses unless they were absolutely certain of the correctness of the response. That is, it is possible that participants were aware of the flankers' identities in Miller's experiment but had adopted such a strict response criterion that they chose not to report the flankers.

Miller (1987, Experiment 4) reported only $16 \%, 21 \%$, and $15 \%$ flanker recall across three conditions. (Note: Although Miller did not report what chance recall would be, we assume that these values are at or near chance). In fact, across the three groups, fewer than 20 letters were named by the 25 participants in each group, an average of less than 1 per participant when six flankers were presented. The low number of reported flankers (correct or not) suggests that participants were employing a strict response criterion. By requiring our participants to report 4 letters that they believed had appeared in the flanker positions, and by legitimizing the correct report of those letters, we minimized response criterion problems relative to Miller's procedure: Participants gave responses even though they may not have been completely confident about the accuracy of their responses and even though they had been instructed to ignore the flankers.

In Experiment 2, we examined the effect of recall procedure on the level of flanker recall by requesting flanker recall twice. The first recall inquiry, which we will refer to as open-ended recall, was modeled after Miller's Experiment 4: The participants were merely asked if they remembered the letters that served as flankers. As noted above, this question, in conjunction with instructions to ignore the flankers, may lead participants to adopt a strict response criterion. Thus, we predicted that open-ended flanker recall would be low, similar to the flanker recall reported by Miller (1987). The second recall inquiry, which we will refer to as forced recall, was modeled after Experiment 1: Immediately following open-ended recall, recall was legitimized, and the participants were required to make a four-letter response. To the extent that a strict response criterion was responsible for the lack of flanker recall reported by Miller (1987), we expected to find low flanker recall after open-ended recall and high flanker recall (mirroring Experiment 1) after forced recall.

\section{Method}

Participants. Twenty-six students ( 13 female and 13 male) received extra credit in an undergraduate psychology course for voluntary participation in this experiment.

Stimuli. The stimuli were nearly identical to those in Experiment 1 . The one difference was that only the redundant flanker condition was used; that is, the same flanker letter was presented to the right and left of the target.

Procedure. The procedure was patterned after that of Experiment 1 , except for changes in how flanker recall and knowledge of the flanker-response correlations were assessed. Stimulus presentation was identical to that of Experiment 1, except that the location cue $\left({ }^{\wedge}\right)$ was only presented during the "READY" display. Two addi- 
Table 3

Mean Latency (in Milliseconds) With Standard Deviations and Proportions Correct for Valid and Invalid Trials and Flanker Effect for Experiments 2 and 3

\begin{tabular}{lccccccc}
\hline & \multicolumn{3}{c}{ Experiment 2} & & \multicolumn{3}{c}{ Experiment 3 } \\
\cline { 2 - 4 } \cline { 6 - 7 } \multicolumn{1}{c}{ Validity } & $M$ & $S D$ & PC & & $M$ & $S D$ & PC \\
\hline Valid & 551 & 124 & .97 & & 508 & 78 & .96 \\
Invalid & 561 & 127 & .97 & & 521 & 84 & .95 \\
Flanker effect & 10 & 20 & & 13 & 24 & \\
\hline
\end{tabular}

Note-Flanker $=$ invalid latency - valid latency.

tional differences were that stimulus displays were presented for only $500 \mathrm{msec}$ (though the response deadline remained $1,500 \mathrm{msec}$ ) and responses were made unimanually by pressing the " $M$ " key with the right middle finger and the " $N$ " key with the right index finger. All other aspects of the procedure mirrored Experiment 1 .

The first recall inquiry, open-ended recall, was designed to replicate that of Miller (1987, Experiment 4). It consisted of the following question: "Can you name all the letters that appeared in the left and right positions of the rows of letters you were presented?" Participants were not required to actually name letters at this point; that is, "I don't know" or "No" was an acceptable response. In addition, an open-ended question about whether or not the participant had noticed any association between certain flankers and certain responses was included. The open-ended flanker recall procedure was followed by a second recall inquiry-forced recall--which was like the recall procedure used in Experiment 1. Participants were required to report four letters that they believed had served as flankers.

Following the experimental trials and the open-ended and forced recall procedures, a sixth block of 32 trials was included to assess participants' implicit memory for the flanker-response correlations. Flankers from the experimental trials were presented, for $40 \mathrm{msec}$, with a blank space in place of a target. After the $40 \mathrm{msec}$, the blank was replaced by an $X$. The $X$ and flankers were displayed until the participant made a response. The participants were instructed that a target letter, which they might not be able to see, would be briefly presented before the $X$ and that they should make the appropriate response. They were told to just make the response that seemed correct when they were unsure about the identity of the target. The cover story indicated that practice responding to targets can sometimes influence how well people perceive the same targets when they are presented briefly. That is, the instructions were designed to make the participants believe that a target was actually presented, so that there would be no explicit attempt to use the flankers during this block of trials. Thus, the extent to which the participants used the flankers to guide their responses could reflect implicit knowledge of the flanker-response correlations.

Immediately following block six, the participants' explicit knowledge of the flanker-response correlations was assessed via two measures. First, flanker assignment was assessed by asking the participants which two flankers they thought usually appeared with the index and middle targets. Second, the participants then completed a recognition test in which all eight index-response displays and all eight middle-response displays were printed in a random order. The participants were asked to circle the four index and four middle displays that they believed had been presented "most often" during the experimental trials (in effect, the instructions were to circle the valid displays; the proportion of correct responses defined valid display recognition).

\section{Results}

An alpha level of .05 was used for all analyses. The data from 2 participants were replaced because their accuracy fell below our $85 \%$ criterion.
Flanker effect. The latency analysis included accurate response latencies greater than $50 \mathrm{msec}$. The mean latencies, standard deviations, and accuracies for valid and invalid trials and the flanker effect are shown in Table 3. The validity difference was reliable [i.e., there was a flanker effect; $t(25)=2.42, S E=3.910$.]

Flanker recall. The mean proportions of flankers correctly recalled using the open-ended and forced recall procedures were calculated by dividing the number of flankers correctly identified by four, the number of flankers to be identified. The proportions are shown in Table 4. As in Experiment 1, chance recall was .18.

Although flanker recall was higher for forced recall than for open-ended recall $[t(25)=2.20, S E=0.052$ ], flanker recall was above chance for both recall procedures $[t(25)=10.02, S E=0.062$, for open-ended recall, and $t(25)=14.54, S E=0.051$, for forced recall] .

Assessment of flanker-response correlations. Knowledge of the flanker-response correlations was assessed in four ways: through the open-ended question that preceded forced recall, through flanker assignment, through valid display recognition, and through implicit use of the flankers during block six. When asked the open-ended question about whether they had noticed any association between certain flankers and certain responses, 12 of 26 participants reported that they had not. The remaining 14 participants reported 31 flanker-response relationships (average 2.2 relationships per participant) of which only 18 were correct. That is, responses to the open-ended flanker assignment question yielded little evidence that the participants were aware of the flanker-response correlations. The mean proportion of correct responses for the remaining three measures is shown in Table 5. Flanker assignment was calculated as in Experiment 1. Valid display recognition was calculated by dividing the total number of valid displays circled by eight, the total number of valid displays. Finally, implicit use of the flankers was calculated as the proportion of correlation-consistent (correct) responses made to block six trials, which contained only the X (in the target position) and the flankers. That is, a correct response was one that reflected the flanker-response correlation.

For all three measures, chance performance was $\mathbf{5 0 .}$ One-tailed tests against chance showed no evidence of above chance performance with any measure of knowledge of the flanker-response correlation $[t(25)=1.16, S E=$ 0.061 , for flanker assignment; $t(25)=1.51, S E=0.038$, for valid display recognition; and $t(25)=1.67, S E=0.036$, for block six].

Table 4

Mean Proportions Correct With Standard Deviation for the Two Types of Flanker Recall in Experiments 2 and 3

\begin{tabular}{cccccc}
\hline & \multicolumn{2}{c}{ Experiment 2 } & & \multicolumn{2}{c}{ Experiment 3 } \\
\cline { 2 - 3 } \cline { 5 - 6 } Type of Recall & $M$ & $S D$ & & $M$ & $S D$ \\
\hline Open-ended & .63 & .32 & .35 & .22 \\
Forced & .74 & .26 & .59 & .23 \\
\hline
\end{tabular}


Table 5

Mean Proportions Correct Flanker Assignment, Valid Display Recognition, and Implicit Use of the Flankers in Block 6 in Experiments 2 and 3

\begin{tabular}{llllll}
\hline & \multicolumn{2}{c}{ Experiment 2 } & & \multicolumn{2}{c}{ Experiment 3 } \\
\cline { 2 - 3 } \cline { 5 - 6 } Variable & $M$ & $S D$ & & $M$ & $S D$ \\
\hline Flanker assignment & .57 & .31 & & .60 & .31 \\
Display recognition & .56 & .19 & .54 & .21 \\
Block 6 & .56 & .18 & .52 & .14 \\
\hline
\end{tabular}

Note-Chance is .50 for each variable.

\section{Discussion}

Experiment 2 was designed primarily to determine whether the different recall results reported in Experiment 1 and Miller's (1987) Experiment 4 were due to differences in the recall procedures. Specifically, we thought that the high flanker recall of Experiment 1, which suggests a failure of selective attention at some point during processing in the correlated flanker task, might be the result a greater degree of sensitivity in our method of assessing flanker recall: We legitimized flanker recall and required participants to report four flanker letters, whereas Miller did not. The results of Experiment 2, in which a Millerlike open-ended recall procedure was followed by the forced recall procedure of Experiment 1, offered some support for this hypothesis. Flanker recall was higher with forced recall than with open-ended recall. However, flanker recall was well above chance even with open-ended recall. Thus, in two experiments, we failed to replicate Miller's finding of no reliable recall. Experiment 3 was another attempt to replicate Miller's finding by changing the nature of the experimental trials.

\section{EXPERIMENT 3}

Although the results of Experiment 2 demonstrated the replicability of high flanker recall in the correlated flanker task and thus lend further support to the idea that there is a failure of selective attention at some point during processing, it was puzzling that we were unable to replicate Miller's (1987) finding of no flanker recall. A closer examination of the procedure in Miller's Experiment 4, however, revealed a major procedural difference between that experiment and ours. In Miller's Experiment 4, a hybrid Eriksen/correlated flanker task was employed in which Eriksen trials were intermixed with correlated trials. That is, in addition to the valid and invalid correlated trials, there were also trials in which target letters were presented in the flanker locations. Miller's Experiment 4 was the only experiment in the six-experiment paper in which flanker recall was assessed, and it was the only experiment in that paper (or in the extant literature, to our knowledge) in which Eriksen and correlated trials were intermixed. Although it was not clear why such a manipulation would adversely affect flanker recall, to the extent that it could, it might explain our failure to replicate Miller's lack of flanker recall.

In Experiment 3, we investigated the effect of using a hybrid flanker task on flanker recall. Essentially, we repli- cated Experiment 2 with the addition of intermixed Eriksen trials. Experiment 3 was designed to examine whether the addition of the Eriksen trials would lead to a replication of Miller's recall results.

\section{Method}

Participants. Twenty-eight students ( 16 female and 12 male) received extra credit in an undergraduate psychology course for voluntary participation in this experiment.

Stimuli. The stimuli for the correlated flanker trials were identical to those used in Experiment 2. The correlated flanker trials were intermixed with Eriksen trials. There were three types of Eriksen trials: identical, compatible, and incompatible. Identical trials were those in which the target letter was presented in all three stimulus locations (e.g., P P P). Compatible trials were those in which the target was surrounded by a letter from the same response set (i.e., the other target from that response set). Incompatible trials were those in which a target was surrounded by letters from the other response set. In the case of the identical and compatible trials, the letters serving as flankers were directly associated with the same response set as was the target, whereas in the case of incompatible trials, the letters serving as flankers were directly associated with the other response set. The typical finding with Eriksen trials is that the response latency is faster for identical and compatible trials than for incompatible trials.

Procedure. The procedure was nearly identical to that of Experiment 2. The first block, which was practice, consisted of 32 validcorrelated trials. The second through fifth blocks were the experimental blocks, in which correlated trials and Eriksen trials were randomly intermixed. Each experimental block consisted of 56 trials of which 32 were valid-correlated trials, 8 were invalid-correlated trials (so flanker validity on these trials remained at .80 but overall flankers were associated with the same response as the target on only .71 of the trials), 4 were identical-Eriksen trials, 4 were compatibleEriksen trials, and 8 were incompatible-Eriksen trials. Thus, the Eriksen trials comprised $29 \%$ of the total trials in each block, just as they did in Miller's (1987) Experiment 2. There were 224 experimental trials total.

Assessment of recall was the same as in Experiment 2, except that the second recall inquiry was prefaced by a request that participants not report target letters as flankers (even though they had indeed appeared in the flanker locations). Flanker assignment, valid display recognition, and use of the flankers in block six were assessed in the same manner as that described for Experiment 2.

\section{Results}

An alpha level of .05 was used for all analyses. The data from 2 participants were replaced because their accuracy failed to meet our $85 \%$ criterion.

Flanker effect. The latency analysis included accurate response latencies greater than $50 \mathrm{msec}$. The mean latencies, standard deviations, and accuracies for valid and invalid trials and the flanker effect are shown in Table 3. The validity difference was reliable [i.e., there was a flanker effect; $t(27)=2.95, S E=4.603$ ] .

The mean accuracies and latencies for the three types of Eriksen trials were: $.97,515 \mathrm{msec}(S D=95.1)$ for the identical trials; $.97,508 \mathrm{msec}(S D=83.1)$ for the compatible trials; and .92, $538 \mathrm{msec}(S D=79.3)$ for the incompatible trials. An ANOVA of the mean latencies for the Eriksen trials showed that the trial types did differ $[F(2,54)=10.93$, $\left.M S_{\mathrm{e}}=642\right]$. Comparisons among the three means using Tukey's HSD showed the pattern typically found with the 
Table 6

Cross-Experiment Correlations of Dependent Measures

\begin{tabular}{lccccc}
\hline & 1 & 2 & 3 & 4 & 5 \\
\hline 1. Flanker effect & - & $.31^{*}$ & .11 & -.01 & -.05 \\
2. Forced recall & & - & $.44^{\dagger}$ & .13 & $.29^{*}$ \\
3. Flanker assignment & & & - & $.58^{\dagger}$ & $.54^{\dagger}$ \\
4. Display recognition & & & & - & $.39^{\dagger}$ \\
5. Block 6 & & & & & -
\end{tabular}

${ }^{*} p<.05 . \quad{ }^{\dagger} p<.01$.

Eriksen flanker task: The latency was longer to incompatible trials than to either the identical trials or the compatible trials, which did not reliably differ (HSD $=16.9)$.

Flanker recall. The mean proportion of the Eriksen flankers (i.e., the targets as flankers) correctly recalled was . 80 (approximately three out of four). The mean proportions of correlated flankers correctly recalled in the open-ended and forced recall procedures were calculated as described in Experiment 2. The proportions are shown in Table 4. Chance recall was .18.

As in Experiment 2, although flanker recall was higher for forced recall than for open-ended recall $[t(27)=4.04$, $S E=0.060$ ], flanker recall was above chance for both recall procedures $[t(27)=9.50, S E=0.043$, for forced recall, and $t(27)=4.07, S E=0.041$, for open-ended recall].

Although flanker recall was above chance in Experiment 3 and there was a higher level of recall with the forced than with the open-ended procedure, it appeared that recall was lower in Experiment 3 than in Experiment 2. A post hoc ANOVA of recall with experiment as a between-subjects variable and type of recall test as a within-subjects variable showed a main effect of experiment, with higher recall in Experiment 2 than in Experiment $3\left[F(1,52)=11.82, M S_{\mathrm{e}}=0.104\right]$, a main effect of recall procedure, with higher recall in the forced recall procedure than in the open-ended procedure $[F(1,52)=$ 30.05, $M S_{\mathrm{e}}=0.029$ ], and a marginally reliable interaction $\left[F(1,52)=3.74, M S_{\mathrm{e}}=0.029, .05<p<.10\right]$, showing a larger negative impact of the Eriksen trials with openended recall.

Assessment of flanker-response correlations. As in Experiment 2, knowledge of the flanker-response correlations was assessed via flanker assignment, valid display recognition, and implicit use of the flankers during block six. The mean proportions of correct responses for all three measures are shown in Table 5.

For all three measures, chance performance was .50 . One-tailed tests against chance showed no evidence of above chance performance with any measure of knowledge of the flanker-response correlation $[t(27)=1.73, S E=$ 0.058 , for flanker assignment; $t(27)=0.91, S E=0.039$, for valid display recognition; and $t(27)=0.70, S E=0.026$, for block six].

Cross-experiment correlations. The data from Experiments 2 and 3 were combined in order to compute correlations among the various dependent measures: the flanker effect, forced flanker recall, flanker assignment, valid display recognition, and implicit use of flankers in block six. Among the 10 correlations, 6 were reliable (see Table 6). As might be expected if there was a failure of selective attention, higher flanker recall was associated with larger flanker effects, more accurate flanker assignment, and even more implicit use of the flankers in block six. However, while accurate flanker assignment was also related to valid display recognition and implicit use of flankers in block six, there was no reliable relationship between flanker assignment and the flanker effect.

\section{Discussion}

Correlated flanker recall was lower in Experiment 3 than it was in Experiments 1 and 2, especially in the openended recall procedure, which was designed to replicate the procedure used by Miller (1987, Experiment 4). It appears as though open-ended recall, which may lead to a relatively strict response criterion, in combination with a hybrid task that includes intermixed Eriksen and correlated trials, can lead to lower correlated flanker recall performance, somewhat like that reported by Miller (1987). However, even under these conditions, we were unable to completely eliminate open-ended flanker recall.

Just why the addition of the Eriksen trials in Experiment 3 led to lower correlated flanker recall, especially when recall was assessed in an open-ended fashion, is unknown. Possibly there is a limit to the number of letters that will be recalled in response to an open-ended question. There were more flankers to remember in the hybrid task (eight in the hybrid task of Experiment 3 as opposed to four in the strictly correlated tasks in Experiments 1 and 2 ), and participants actually recalled similar overall proportions of flankers (Eriksen and correlated) in response to the open-ended question in Experiment $3(.58)$ and Experiment 2 (.63); but the Eriksen trials affected which letters were reported.

The Eriksen flankers are strongly represented in memory. These strong representations might interfere with the accessibility of weaker memories of the correlated flankers' identities (cf. the output interference literature; Roediger \& Schmidt, 1980). Given that the Eriksen flankers are so well remembered and the fact that recall does go up between the open-ended and forced recall procedures, the lower overall recall performance found in Experiment 3 openended recall and in Miller (1987, Experiment 4) likely reflects lack of accessibility to rather than availability of representations of all the flankers (cf. Tulving \& Pearlstone, 1966).

Recall of the correlated flankers in Experiment 3 also could be lower as the result of some form of inhibition. The processing of flankers in a standard Eriksen flanker task may have to be actively inhibited to prevent making the wrong response. As pointed out to us by a reviewer, this active inhibition might not develop in the correlated flanker task. If it develops to some extent in the hybrid task, the inhibition might affect accessibility of the flanker memory traces.

Although the present findings suggest that flanker recall can be reduced by using a hybrid task, the hybrid task 
is not common in the literature. In the typical correlated flanker task (cf. Carlson \& Flowers, 1996; Miller, 1991; Paquet \& Lortie, 1990), there are no Eriksen trials. Thus, the recall data from Experiments 1 and 2 should be more indicative of the type of processing (in a correlated flanker task) represented in the literature. The data indicate that there is a failure of selective attention at some point during processing in the typical correlated flanker task.

The failure of the selective attention interpretation of the flanker effect suggests that the flanker effect results from attentional processing of the flankers, and the data show that flankers do receive attentional processing. It is unclear whether the failure of selective attention hypothesis implies anything more than that, but the data speak to one further implication. Although the flanker effect is associated with knowledge of the flanker identities, as shown by the high flanker recall in all conditions and the reliable correlation between the magnitude of the flanker effect and the number of flankers recalled collapsed over Experiments 2 and 3, it does not depend on one's explicitly knowing the relationship between the flankers and the responses. Neither Miller's (1987) participants nor ours showed any indication that they were aware of the exact flanker-response relationships present in the displays (see also Carlson \& Flowers, 1996). The flanker effect appears to depend on some implicit, rather than explicit, representation of the flanker-response correlations.

\section{GENERAL DISCUSSION}

We began the present research by examining the extent to which flanker redundancy might contribute to the flanker effect. Although the results did not implicate flanker redundancy as an important variable, the high level of flanker recall suggested that flankers had received attentional processing; that is, there was a failure of selective attention at some point during processing in the correlated flanker task. The recall data were in direct contrast with the only other flanker recall data of which we were aware (Miller, 1987, Experiment 4). The subsequent two experiments were designed to untangle the reasons for the conflicting outcomes. In both experiments, flanker recall was high. Thus, in all conditions across three experiments, the participants were quite able to recall what letters had served as flankers.

Recall is a measure of explicit memory, and it therefore depends on attentional rather than automatic processing (e.g., Eich, 1984; Fisk \& Schneider, 1984; Hasher \& Zacks, 1979; Rock \& Gutman, 1981; Roediger, 1990; Shiffrin \& Schneider, 1977). Thus, the recall data show that flankers were not excluded from attentional processing. Only when an unusual hybrid task and a less sensitive recall measure were used was flanker recall anywhere near the chance level that would be expected if flankers received only automatic processing. The fact that a subsequent forced recall procedure produced an increase in the level of recall shows that the information about flanker identity was available in the system even though it might not have been accessible (see Tulving \& Pearlstone, 1966).
Three major points can be drawn from the present research. The first concerns the relationship between intention and attention. The failure of selective attention found here suggests that intention alone cannot always restrict attention to relevant stimuli as assumed by the intentionequals-attention view of processing. We assume that participants were attempting to follow instructions to attend to the targets and to ignore the flankers, but that they were unable to do so. The idea that intention cannot completely restrict attention deserves further discussion, because in some research, intention has been used as a measure of attention. That is, when the experimenter instructs the participant to "attend" to $X$ and "ignore" $Y$, it is assumed that only $\mathrm{X}$ receives attentional processing and that any characteristics of to-be-ignored $Y$ that influence $X$ must reflect automatic processing. Our data suggest that equating intention with attention is not theoretically justified, because unattended versus attended refers to the kind of processing (automatic or attentional) that a stimulus receives. From a dual-process approach, it is possible for a to-beignored stimulus to be attended; it depends on what kind of processing, automatic or attentional, the stimulus receives. Some converging evidence that a stimulus has or has not been attended is needed to determine whether the instructions have been followed successfully. We used flanker recall as such a measure, because recall reflects explicit memory for attentionally processed stimuli.

The second point concerns the theoretical interpretation of the flanker effect. The high level of flanker recall found in the present research is inconsistent with the past interpretations of the flanker effect that have suggested that the flankers are unattended (Miller, 1987; Shiffrin et al., 1996). It is clear that, at least under display conditions similar to those employed here, which are quite typical of the conditions employed in the bulk of the literature, participants do become aware of the flankers' identities. These findings have serious implications for using the flanker task to address the nature of automatic processing. That is, these findings suggest that there is a failure of selective attention in the correlated flanker task such that the flankers are attended, not unattended.

There is, however, an interpretation of the recall findings that would allow the flanker effect to be the result of processing of unattended flankers. Scheerhorn and Dark (1995) called this interpretation the broadening of attention hypothesis. According to this hypothesis, attentional processing of the flankers (i.e., the failure of selective attention) occurs after target processing is complete. Attention is focused only on the target until target processing is complete and response selection has occurred. After response selection, the focus of attention broadens to include the flankers. Thus, flanker recall could reflect attentional processing of the flankers in the interval between response selection and the termination of the stimulus display, but the flanker effect would be the result of automatic processing of the flankers before response selection.

In examining this hypothesis, Scheerhorn and Dark (1995) manipulated flanker duration so that the flankers disappeared from the stimulus display after 500, 400,300, 
200 , or $100 \mathrm{msec}$. They reasoned that unless response selection was completed before $100 \mathrm{msec}$, the broadening of attention hypothesis would predict that some condition or conditions should show a flanker effect without high flanker recall. In fact, flanker recall was above chance in all five duration conditions. Scheerhorn and Dark (1995) concluded that the findings suggested a broad focus of attention beginning at stimulus onset rather than a narrow focus of attention that became broad after response selection.

Scheerhorn and Dark (1995) manipulated flanker display duration in an attempt to limit attentional processing of flankers. However, there is still another interpretation of the situation that allows flanker recall to be the result of attentional processing after response selection: Flanker recall could be the result of attentional processing of a memory trace (of the flankers). Thus, even when a high level of recall is obtained, it is not conclusive evidence that the failure of selective attention occurs before response selection. The recall data show only that there is a failure of selective attention at some point during processing in the correlated flanker task.

Miller (1987) developed the correlated flanker task partly in response to challenges to the interpretation of Eriksen flanker data in terms of the nature of processing of unattended stimuli (e.g., Johnston \& Dark, 1986; Kahneman \& Treisman, 1984). The present research suggests that there is attentional processing of flankers in the correlated flanker task, but it is unclear just when the attentional processing occurs. It appears that the correlated flanker task also cannot be used to unambiguously investigate the nature of attentional as opposed to automatic processing.

The third and final point concerns the relationship between the flanker effect and explicit knowledge. As pointed out by Miller (1987), the flanker effect is not the result of intentional use of a known relationship between the flankers and the responses. The flanker effect appears to reflect incidental learning of a relationship between attended stimuli. Although the flanker recall data show that the flankers were attended in the correlated flanker task, the flanker assignment data show that most participants were unaware of the flanker-response correlations. The participants were influenced by the flanker correlationthat is, they showed flanker effects in all conditions-but there was little evidence that they were consciously aware of what was influencing them or of the fact that they were being influenced (cf. Lewicki, Hill, \& Czyzewska, 1992; Nisbett \& Wilson, 1977).

It appears that attentional processing of correlated events can, at least sometimes, lead to the learning of relationships that cannot be consciously described. The flanker-response correlations is a simple association between contiguous events (the display and the response), and there is evidence that such associations can be learned without attentional processing of the relationship itself (e.g., Curran \& Keele, 1993; Lambert \& Sumich, 1996; Stadler, 1992). Recent studies by Carlson and Flowers (1996) have demonstrated the unintentional use of simple contingency information by participants who had and had not been informed of the contingency. These findings suggest that although attentional processes may be required in order to learn contingencies, some of that learning may be "automatic" in that it occurs without intention and without explicit awareness of what is being learned. This is not the same, however, as learning about relationships involving stimuli of which the participant is unaware as Miller (1987) originally suggested. There is a failure of selective attention at some point during processing in the correlated flanker task, and participants are aware of the flanker identities; but they are not aware of the flanker-response correlation.

\section{REFERENCES}

BAYLIS, G. C., \& DrIVER, J. (1992). Visual parsing and response competition: The effect of grouping factors. Perception \& Psychophysics, 51, 145-162.

Broadbent, D. E. (1958). Perception and communication. London: Pergamon.

CARLSON, K. A., \& Flowers, J. H. (1996). Intentional versus unintentional use of contingencies between perceptual events. Perception \& Psychophysics, 58, 460-470.

Chastain, G., Cheal, M., \& Lyon, D. R. (1996). Attention and nontarget effects in the location-cuing paradigm. Perception \& Psychophysics, 58, 300-309.

CoWAN, N. (1988). Evolving conceptions of memory storage, selective attention, and their mutual constraints within the human informationprocessing system. Psychological Bulletin, 104, 163-191.

Curran, T., \& KEElE, S. W. (1993). Attentional and nonattentional forms of sequence learning. Journal of Experimental Psychology: Learning, Memory, \& Cognition, 19, 189-202.

Deutsch, J. A., \& Deutsch, D. (1963). Attention: Some theoretical considerations. Psychological Review, 70, 80-90.

EICH, E. (1984). Memory for unattended events: Remembering with and without awareness. Memory \& Cognition, 12, 105-111.

ERIKSEN, B. A., \& ERIKSEN, C. W. (1974). Effects of noise letters upon the identification of a target letter in a nonsearch task. Perception \& Psychophysics, 16, 143-149.

ERIKSEN, C. W., \& HoffMan, J. E. (1973). The extent of processing of noise elements during selective encoding from visual displays. Perception \& Psychophysics, 14, 155-160.

ERIKSEN, C. W., \& Schultz, D. W. (1979). Information processing in visual search: A continuous flow conception and experimental results. Perception \& Psychophysics, 25, 249-263.

Evans, P. M., \& Craig, J. C. (1992). Response competition: A major source of interference in a tactile identification task. Perception \& Psychophysics, 51, 199-206.

FisK, A. D., \& SCHNEIDER, W. (1984). Memory as a function of attention, level of processing, and automatization. Journal of Experimental Psychology: Learning, Memory, \& Cognition, 10, 181-197.

Flowers, J. H. (1990). Priming effects in perceptual classification. Perception \& Psychophysics, 47, 135-148.

FLOWERS, J. H., \& WILCOX, N. (1982). The effect of flanking context on visual classification: The joint contribution of interactions at different processing levels. Perception \& Psychophysics, 32, 581-591.

Fournier, L. R., \& ErIKsEn, C. W. (1990). Coactivation in the perception of redundant targets. Journal of Experimental Psychology: Human Perception \& Performance, 16, 538-550.

HARMS, L., \& BUNDESEN, C. (1983). Color segregation and selective attention in a nonsearch task. Perception \& Psychophysics, 33, 11-19.

HASHER, L., \& ZACKS, R. T. (1979). Automatic and effortful processes in memory. Journal of Experimental Psychology: General, 108, 356-388.

Hoffman, J. E. (1979). A two-stage model of visual search. Perception \& Psychophysics, 25, 319-327.

HOLENDER, D. (1986). Semantic activation without conscious identification in dichotic listening, parafoveal vision, and visual masking: A survey and appraisal. Behavioral \& Brain Sciences, 9, 1-23. 
Johnston, W. A., \& DARK, V. J. (1986). Selective attention. Annual Review of Psychology, 37, 43-75.

JoNIDES, J. (1981). Voluntary versus automatic control over the mind's eye's movement. In J. B. Long \& A. D. Baddeley (Eds.), Attention and performance IX (pp, 187-203). Hillsdale, NJ: Erlbaum.

Kahneman, D., \& Treisman, A. (1984). Changing views of attention and automaticity. In R. Parasuraman \& D. R. Davies (Eds.), Varieties of attention (pp. 29-61). New York: Academic Press.

Kramer, A. F., \& JaCOBSON, A. (1991). Perceptual organization and focused attention: The role of objects and proximity in visual processing. Perception \& Psychophysics, 50, 267-284.

LAMBERT, A. J. (1985, Fall). Selectivity and stages of processing-An enduring controversy in attentional theory: A review. Current Psychological Research \& Reviews, pp. 239-256.

LAMBerT, A. J., \& Sumich, A. L. (1996). Spatial orienting controlled without awareness: A semantically based implicit learning effect. Quarterly Journal of Experimental Psychology, 49, 490-518.

LEWICKI, P., Hill, T., \& CzyzewSKA, M. (1992). Nonconscious acquisition of information. American Psychologist, 47, 796-801.

Logan, G. D. (1988). Toward an instance theory of automatization. Psychological Review, 95, 492-527.

LOGAN, G. D. (1995). Linguistic and conceptual control of visual spatial attention. Cognitive Psychology, 28, 103-174.

McCANN, R. S., Folk, C. L., \& Johnston, J. C. (1992). The role of spatial attention in visual word processing. Journal of Experimental Psychology: Human Perception \& Performance, 18, 1015-1029.

MILLER, J. (1982). Divided attention: Evidence for coactivation with redundant signals. Cognitive Psychology, 14, 247-279.

Miller, J. (1987). Priming is not necessary for selective-attention failures: Semantic effects of unattended, unprimed letters. Perception \& Psychophysics, 41, 419-434.

MILLER, J. (1991). The flanker compatibility effect as a function of visual angle, attentional focus, visual transients, and perceptual load: A search for boundary conditions. Perception \& Psychophysics, 49, 270-288.

NeumanN, O. (1984). Automatic processing: A review of recent findings and a plea for an old theory. In W. Prinz \& A. F. Sanders (Eds.) Cognition and motor processes (pp. 255-293). Berlin: Springer-Verlag.

NisBetT, R. E., \& WiLson, T. D. (1977). Telling more than we know: Verbal reports on mental processes. Psychological Review, 84, 231-279.

Paquet, L., \& Lortie, C. (1990). Evidence for early selection: Precuing target location reduces interference from same-category distractors. Perception \& Psychophysics, 48, 382-388.

POSNER, M. I., \& SNYDER, C. R. R. (1975). Attention and cognitive control. In R. L. Solso (Ed.), Information processing and cognition: The Loyola Symposium (pp. 55-85). Hillsdale, NJ: Erlbaum.

Rock, I., \& GUTMaN, D. (1981). The effect of inattention on form perception. Journal of Experimental Psychology: Human Perception \& Performance, 7, 275-285.

ROEDIGER, H. L., III (1990). Implicit memory: Retention without remembering. American Psychologist, 45, 1043-1056.

RoEDIGER, H. L., III, \& SCHMIDT, S. R. (1980). Interference in the recall of categorized and paired-associate lists. Journal of Experimental Psychology: Human Learning \& Memory, 6, 91-105.

SCHEERHORN, P. A., \& DARK, V. J. (1994, May). "Unattended" processing of symmetric and asymmetric flankers? Paper presented at the 66th Annual Meeting of the Midwestern Psychological Association, Chicago.

SCHEERHORN, P. A., \& DARK, V. J. (1995, May). Where/when is attention focused in the flanker task? Paper presented at the 67th Annual Meeting of the Midwestern Psychological Association, Chicago.
SCHNEIDER, W. (1988). Micro Experimental Laboratory: An integrated system for IBM PC compatibles. Behavior Research Methods, Instruments, \& Computers, 20, 206-217.

Shiffrin, R. M., Diller, D., \& COHEN, A. (1996). Processing visual information in an unattended location. In A. F. Kramer, M. G. H. Coles, \& G. D. Logan (Eds.) Converging operations in the study of visual selective attention (pp. 225-226). Washington, DC: American Psychological Association.

ShIfFrin, R. M., \& SCHNEIDER, W. (1977). Controlled and automatic human information processing: II. Perceptual learning, automatic attending, and a general theory. Psychological Review, 84, 127-190.

STADLER, M. A. (1992). Statistical structure and implicit serial learning. Journal of Experimental Psychology: Learning, Memory, \& Cognition, 18, 318-327.

Stadler, M. A., \& Proctor R. W. (1993, November). Attention and covariation learning in the Eriksen flanker task. Paper presented at the 34th Annual Meeting of The Psychonomic Society, Washington, DC.

TAYLOR, D. A. (1977). Time course of context effects. Journal of Experimental Psychology: General, 106, 404-426.

Treisman, A. M., \& Gelade, G. (1980). A feature-integration theory of attention. Cognitive Psychology, 12, 97-136.

Tulving, E. (1983). Elements of episodic memory. Oxford: Oxford University Press, Clarendon Press.

Tulving, E., \& Pearlstone, Z. (1966). Availability versus accessibility of information in memory for words. Journal of Verbal Learning \& Verbal Behavior, 5, 381-391.

YANTIS, S., \& JoHNSTON, J. C. (1990). On the locus of visual selection: Evidence from focused attention tasks. Journal of Experimental Psychology: Human Perception \& Performance, 16, 135-149.

\section{NOTES}

1. The logic of this argument requires that the failure of selective attention occur before response selection. However, a reviewer suggested that a failure could occur after response selection. This possibility is addressed in the General Discussion.

2. A programming error in Experiment 1 yielded uninterpretable data in block six.

3. Because there was no reason to assume that performance would be lower than chance, we always used one-tailed tests. We assume that the very low (below-chance) flanker assignment data in the redundant condition are anomalous. The pattern was not replicated in Experiment 2 or 3 or in other studies from our lab.

4. A reviewer suggested that our high flanker recall results, which are in direct opposition to Miller's (1987) recall results, might be due to a procedural difference between the two experiments. Specifically, we employed an initial practice block of 32 trials in which flanker validity was $100 \%$, whereas Miller did not. Perhaps participants "noticed" the usefulness of the flankers during that initial block of trials and attended to them; that is, perhaps the nature of our practice was responsible for the failure of selective attention suggested by our recall results. Although the hypothesis is reasonable, we think it is unlikely that the practice block affected recall. Past research in our lab has shown levels of recall comparable to those of Experiment 1 under conditions in which flanker validity was .50 during practice and throughout the experimental trials (Scheerhorn \& Dark, 1994). Thus, it does not seem as though the correlation per se is what draws attention to the flankers.

(Manuscript received September 20, 1996; revision accepted for publication February 1, 1997.) 